\title{
Controlled Drug Delivery to the Eye: Imaging Mixtures of Drug Polymorphs within Polymer Matrices
}

\author{
K.R. Wormuth and T. M. Anderson \\ SurModics, Inc., 9924 West $74^{\text {th }}$ St., Eden Prairie, MN 55344
}

Diseases of the back of the eye such as macular degeneration or diabetic macular edema require drug delivery deep into the interior of the eye. Since periodic injection of drug via a needle into the eye is unpleasant, one option for the treatment of back of the eye diseases is to implant a monolith or device containing drug encapsulated within a polymer matrix. The polymer matrix is designed to control the rate of release of the drug, and deliver the desired dose of drug over a long time period. Understanding the morphology of drug/polymer mixtures is critical in the design of an effective controlled drug delivery device. Here we study mixtures of the steroid triamcinolone acetonide (TA) blended by various processes with poly(butyl methacrylate) (PBMA) and poly(ethylene-co-vinyl acetate) (PEVA). By altering the morphology of TA/PBMA/PEVA blends, controlled drug delivery for over two years duration is possible, as shown both in vitro and in human in vivo studies.

Depending upon the preparation method, the steroid TA itself can exist in three polymorphic forms: anhydrous crystals, hydrate crystals, and the non-crystalline amorphous form [1]. Under the scanning electron microscope, the anhydrous crystals appear irregularly shaped, the hydrate crystals appear plate-like, and the amorphous form appears glassy. However, upon comminution and dispersion into mixtures of the polymers PBMA and PEVA, identification of the polymorphic form becomes challenging. Microtome cross-sections of dispersions of TA crystals in polymer as imaged in the scanning electron microscope show TA particles are visible within the polymer matrix (Figure 1a).

Although x-ray diffraction can distinguish the crystalline forms of TA, the method is not sensitive to the presence of the amorphous form [2]. On the other hand, scanning confocal Raman microscopy does distinguish between all polymorphic forms of TA, since the Raman spectra of each of the three forms of TA differ significantly (Figure 1b). Raman microscopy, with proper reference materials and deconvolution software, allows imaging of the spatial distribution of the up to five components (three polymorphs of TA and two polymers) possible in TA/PBMA/PEVA mixtures (Figure 2).

One critical question in understanding drug delivery from TA/PBMA/PEVA mixtures is whether the polymorphic state of TA influences the elution rate of the drug from the polymer matrix. As elution proceeds, water is likely absorbed into the polymer and surrounds the drug, crystals of drug dissolve, and drug diffuses through the polymers and out of the matrix. As water is absorbed into the coating, it may change the hydration state of the TA crystals, and thus change the polymorphic form. The dissolution thermodynamics of the anhydrous and hydrate crystalline forms differ in the absence of polymer, and thus may alter drug elution when present in a polymer matrix. These questions will be addressed in the presentation.

References

[1] C. Näther and I. Jeß., Angew. Chem. Int. Ed., 45 (2006) 6381.

[2] R. Hilfiker, ed., Polymorphism in the Pharmaceutical Industry, Wiley-VCH, Weinheim, 2006 

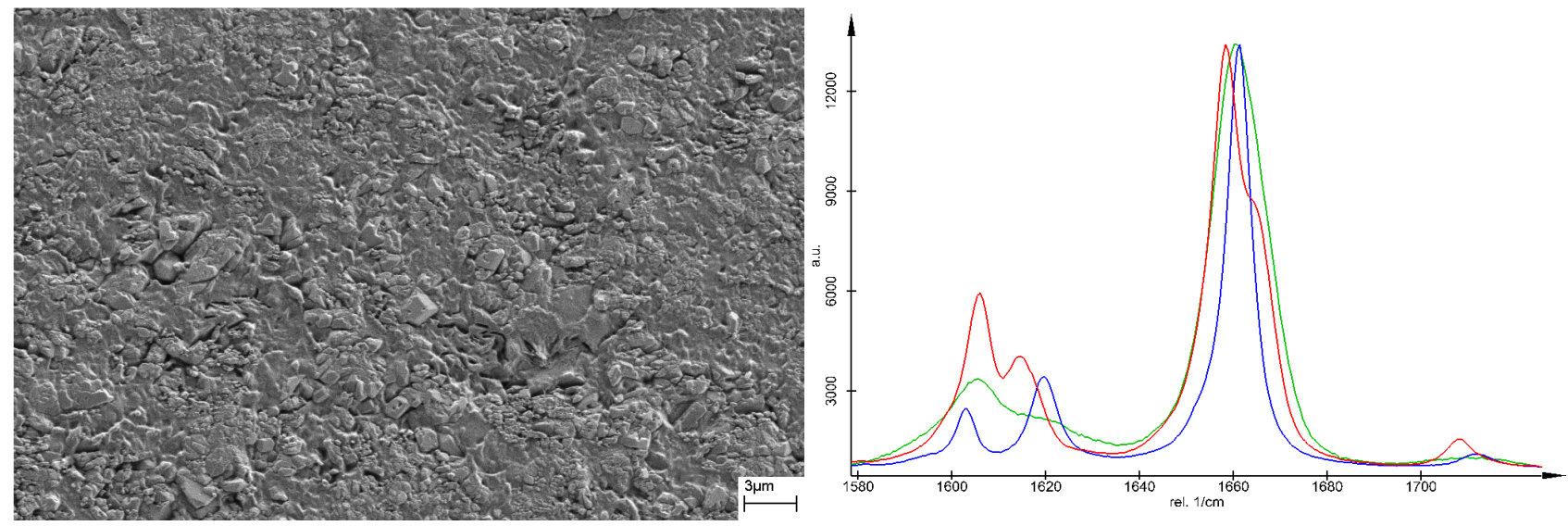

FIG. 1a (Left). SEM image of distribution of TA crystals within PBMA/PEVA polymer matrix

FIG. 1b (Right). Raman spectra of TA polymorphs:

Red $=$ Hydrate, Green $=$ Amorphous, Blue $=$ Anhydrous

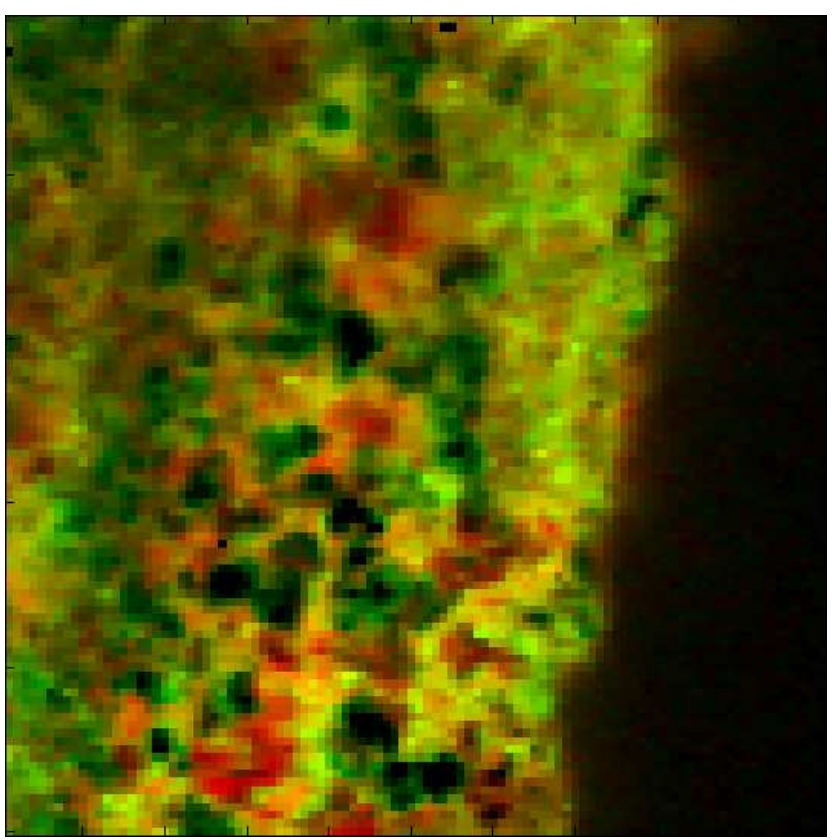

$50 \times 50 \mu \mathrm{m}$

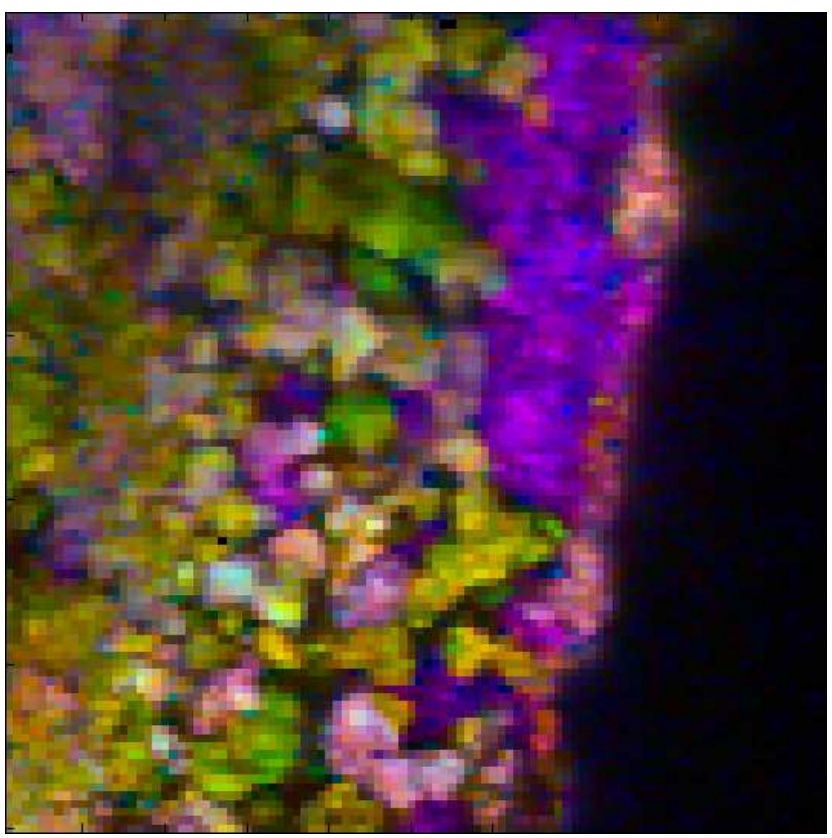

$50 \times 50 \mu \mathrm{m}$

FIG. 2a (Left). Raman image of polymer distribution in a TA/PEVA/PBMA mixture: Red $=$ PBMA, Green $=$ PEVA

FIG. 2 b (Right). Raman image of drug distribution in a TA/PEVA/PBMA mixture: Red $=$ Amorphous TA, Green $=$ Hydrate TA, Blue $=$ Anhydrous TA 